\title{
Non-Newtonian Blood Flow around Healthy and Regurgitated Aortic Valve with Coronary Blood Flow Involved
}

\author{
Jure Marn - Jurij Iljaž* - Zoran Žunič - Primož Ternik \\ University of Maribor, Faculty of Mechanical Engineering, Slovenia
}

Most numerical simulations about aortic valve are discussing about the blood flow through prosthetic valves, therefore this study covers a biological aortic valve. In this paper the simulation of blood flow around the regurgitated valve as well as around the health valve have been investigated and is showing how the regurgitation affects the heart function and coronary blood flow. Furthermore it also covers the coronary blood flow and its affect on the blood motion around the valve and consequently on the possible calcification. The physical problem has been treated for the period of closed valve, because of the regurgitation, which occurs at that time. For this restriction the assumption of non-elastic geometry has been used. Also the non-Newtonian Power Law model for the blood rheology has been used; because of the regions of slowly moving blood. Results are showing that the coronary blood flow does not have a high impact on the blood motion and thus the calcification is more probable as well that the regurgitation has a strong affect on the heart function, like aortic pressure drop, increased left ventricle volume, etc. The calcification reason has also been stated, but needs some further investigation. This paper covers in detail the geometry of computational domain and physics, so the results can be easily repeated.

Keywords: aortic valve, regurgitation, computational fluid dynamics, non-Newtonian

\section{O INTRODUCTION}

An aortic valve is lying between aorta and left ventricle (LV) that pumps the blood through the valve to the aorta and consequently to the whole body. Therefore during the cardiac cycle this valve is heavily stressed and can be marked as one of the most important valves in the heart. For this reason the valve failure is more probable and it will have a significant impact on the cardiac cycle, heart and human health itself. This is the reason why it is so important to understand and study the causes for the valve failure and how they affect the cardiac cycle and therefore human health.

Many numerical models have been developed through time for the purpose to describe the blood flow around prosthetic aortic valve and its effect on it as presented in the study of Lai et al. [1] and Krafczyk et al. [2]. The reason was to investigate the behaviour of the valve and what was the cause of its failure. At first the fluid-structure interaction was ignored because of the numerical complexity, which has been overcome with the work of De Hart et al. [3] to [6] and Carmody et al. [7]. These numerical models have been developed to describe the blood flow around biological and prosthetic aortic valve with major focus to describe the leaflet motion and induced stresses. They do not describe the blood flow around aortic valve when it fails and how this failure affect the cardiac cycle as well they do not include the coronary arteries and the effects on the coronary blood flow, which is the main objective of this study. The blood flow through coronary arteries has already been covered in study of Johnston et al. [8] and [9] and Boutsianis et al. [10] that used only the part of main coronary artery, left or right, without its origin and its aortic pressure dependent blood flow.

We noted the work of De Hart and his coworkers'. They started with two dimensional aspect of the aortic valve involving fluid-structure interaction using Newtonian fluid model, presented in the work [3]. They continue their work by $3 \mathrm{D}$ computational analysis of fluid-structure interaction, still using Newtonian fluid model [4]. The 3D geometry of aortic valve has also been used by Carmody et al. [7]. They take the research of blood flow through aortic valve to the next level, with including the LV and blood flow through it.

The geometric parameters of biological aortic valve treated in this study have been adopted from Labrosse et al. [11] except for sinus depth and diameter of coronary arteries adopted from De Hart et al. [4] and Johnston et al. [8]. The latter work should also be noted for the use of non-Newtonian blood models.

Far more work has been carried out in the area of mechanical heart valves; however mechanical aortic valves were not the subject of our interest.

This paper presents a transient simulation of blood flow around healthy and chronically regurgitated aortic valve including the coronary arteries. Regurgitation of aortic valve is the disease or valve failure when it cannot close completely during its closure and consequently enables the blood to leak back into the left ventricle, while the ejection or valve opening is not problematic. For this reason the 
problem has been treated only for the period of closed valve. The second reason for the chosen time interval is also in the coronary blood flow, which is maximal and has the biggest effect on the blood motion around aortic valve and possible calcification. During the closed valve the valve or its cups movement can be neglected and for this reason the assumption of using non-elastic geometry has been made. Also one important comment has to be made here, that the blood behaves as a non-Newtonian pseudo plastic fluid, especially when the shear rate is small, that is less than $100 \mathrm{~s}^{-1}$. Therefore it is necessary to take this into account, because the blood movement around aortic valve during the closed valve is slow. In this place the Power Law model has been used to describe the properties and behaviour of the blood. The problem was solved with Computational Fluid Dynamics (CFD) using commercial software package.

The aim of this study is to analysis the blood flow around healthy and regurgitated aortic valve during closed valve as well to analysis the effect of the coronary blood flow on the blood around cusps. This work also presents the base for studying the effects of regurgitated aortic valve on the cardiac cycle (aortic pressure drop, regurgitated blood volume, etc.) and on the coronary blood flow.

The remainder of this paper is organized as follows: Section 1 discusses the governing equation for the physical problem; Section 2, the geometry and physics of the problem; Section 3, the results and discussion of this study; and Section 4, conclusions which can be drawn from this work.

\section{GOVERNING EQUATIONS}

Physical problem of blood flow around aortic valve and through coronary arteries can be described mathematically with momentum equation:

$$
\rho \frac{\partial v_{i}}{\partial t}+\rho v_{j} \frac{\partial v_{i}}{\partial x_{j}}=\rho f_{m i}-\frac{\partial p}{\partial x_{i}}+\frac{\partial \tau_{j i}}{\partial x_{j}},
$$

and continuity equation:

$$
\frac{\partial \rho}{\partial t}+\frac{\partial\left(\rho v_{i}\right)}{\partial x_{i}}=0
$$

where $v_{i}$ represents the velocity vector, $p$ static pressure, $\rho$ density, $\tau_{i j}$ the shear stress term and $f_{m i}$ the density of volumetric or mass force. The shear stress term in Eq. (1) is defined with rheological model:

$$
\tau_{i j}=2 \eta \dot{\varepsilon}_{i j}-\frac{2}{3} \eta \dot{\varepsilon}_{k k} \delta_{i j}
$$

where $\eta$ represents the dynamic viscosity, $\dot{\varepsilon}_{i j}$ velocity deformation and $\delta_{i j}$ the Knocker delta function. Rheological model Eq. (3) is valid only for Newtonian fluid; however it is also used to model the nonNewtonian fluid flow. Non-Newtonian behaviour is taken into account with additional model, which connects the dynamic viscosity of the fluid $\eta$ with the shear rate $\dot{\gamma}$ and it will be presented in the next chapter, together with the problem physic and boundary conditions.

Presented system of governing equation, Eqs. (1) to (3) is highly non-linear and therefore solved numerically using the techniques of CFD. As mentioned, the problem has been solved numerically using commercial software package based on the finite volume method (FVM).

\section{NUMERICAL MODEL}

\subsection{Geometry}

Geometry of a computational domain is shown on Fig. 1 and it consists of aortic valve, part of the ascending aorta, part of left ventricle (LV) and origin of both coronary arteries. It can also be seen that small geometric simplifications have been made to simplify the modelling. The reason lies in geometry of the real biological aortic valve, which is not symmetrical as well the ascending aorta does not have a perfect tubular shape. Despite this small simplification the presented geometry in this chapter still represent a normal shape of biological aortic valve and its region.

Aortic valve consists of three major components; annulus, cusp and commissures. The three half-moon shaped (semilunar) cusps are attached to the aortic root and annulus. Between the free and closing edge of each cusp are two areas known as the lunulas. This way the shape of the cusps and the lunulas gives the valve a good sealant property during diastole of LV.

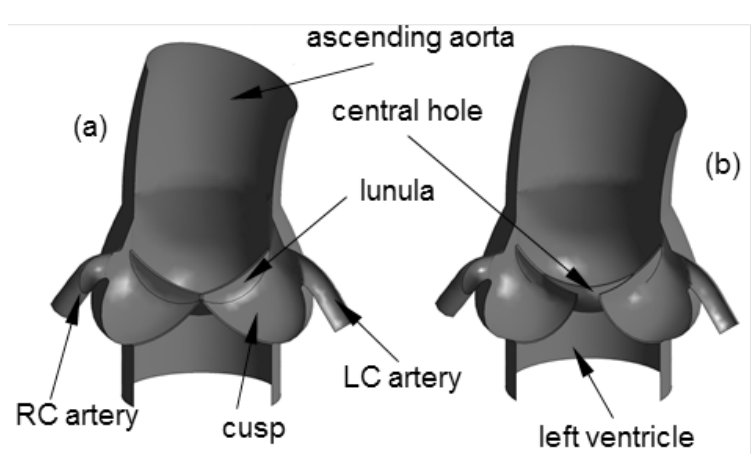

Fig. 1. Geometry of computational domain; a) healthy aortic valve, b) regurgitation 
Ascending aorta arises behind aortic valve and it consists of sinus and tubular portion. Behind the valve cusps there are three sinuses and their main task is to prevent cusps to stick on the aortic wall. From right and left aortic sinus arise the right and left coronary artery as shown on Fig. 1. They supply the blood to the muscular tissue of the heart called myocardium. The right coronary $(\mathrm{RC})$ artery normally supplies the inferior surface of the LV, the right ventricle (RV) and right atrium (RA) in contrast to the left coronary (LC) artery, which supplies the rest of the LV and left atrium (LA).

The important geometric parameters for average biological aortic valve, $\mathrm{LV}$, ascending aorta and coronary arteries are shown on Fig. 2 and gathered in Table 1, together with their values. Introduced geometric parameters have been taken from Labrosse et al. [11] and De Hart et al. [4]. At creating the geometry of the computational domain some assumptions have been made, like equal cusps size, equal diameter of the valve base and sinus tubular junction (STJ) and slightly smaller height of the commissures compared to the sinuses. All these assumptions are not so crucial, because the aortic valve geometry depends on the individual therefore this aortic valve is still representing the normal biological valve.

Coronary arteries parameters, like height of origin and position, have been taken from anatomy [12]. Normally both coronary arteries arise at the two thirds of the sinus height from the aortic annulus to the STJ and near the middle between commissures. RC artery arise nearly perpendicular from the aorta, where LC artery arises at an acute angel. Only parameter that has been taken from Johnston et al. [8] has been the diameter of coronary arteries, which is assumed to be equal.

The tubular portion of ascending aorta was modelled as a slightly curved tube in a direction of the left cusp, with the same diameter as the STJ. Also the part of LV was designed as the tube with base valve diameter, because there has not been enough data to construct the ventricle around aortic valve. In this manner the computational domain for healthy aortic valve has been created.

Computational domain for regurgitated aortic valve has been created in the same way and by the same assumptions. To generate the central hole, which allows the blood to leak back into the LV during the diastole, the base, STJ and aortic diameter have been enlarged from 25 to $27 \mathrm{~mm}$. All other parameters stayed the same. This enlargement created the central hole area of $44.2 \mathrm{~mm}^{2}$, which is $7.7 \%$ of the total valve area. The created geometry of computational domain is shown on Fig. 1.

Table 1. Geometric and Power Law parameters

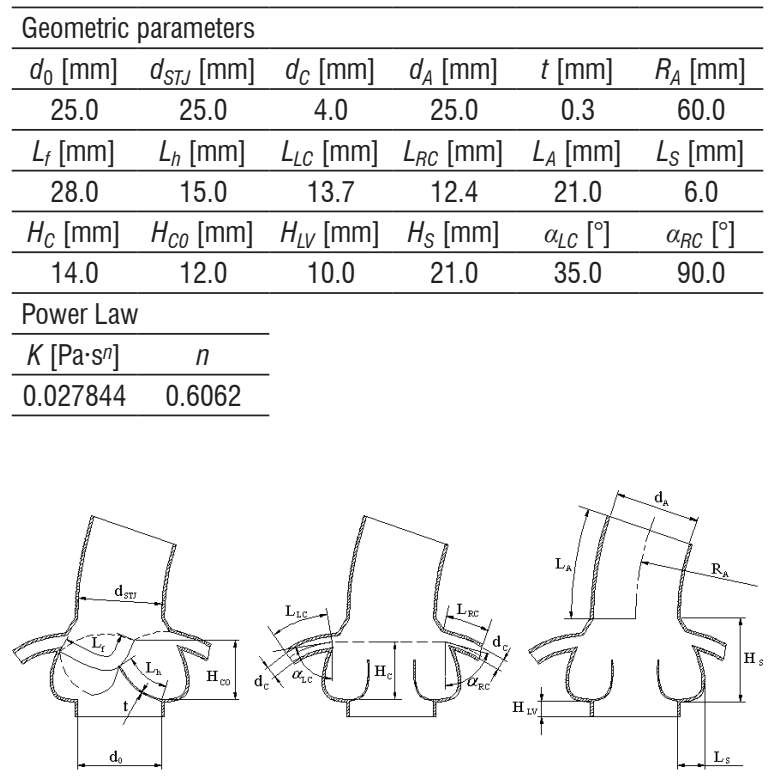

Fig. 2. Important geometric parameters of computational domain

\subsection{Physics}

As mentioned before, the numerical simulation of blood flow around healthy and regurgitated aortic valve, during its closed position, has been done. The deformation of the closed valve and its surrounding region, because of the changing aortic and LV pressure as well as the heart movement is assumed to be small enough; therefore the error with assumption of using non-elastic geometry is small. Although the non-elastic geometry was used, the problem has been still treated as time dependent.

The blood behaves like a pseudo plastic nonNewtonian fluid for which the Power Law model has been used. It correlates the dynamic viscosity of the fluid $\eta$ with theshear rate $\dot{\gamma}$ by equation:

$$
\eta=K \cdot \dot{\gamma}^{n-1},
$$

where and are the Power Law parameters. The values for these parameters have been taken from Ternik et al. [13] and are written in Table 1. When the shear rate is small the dynamic viscosity is high and vice versa. Also the blood has been treated as incompressible fluid, with constant density of $1058 \mathrm{~kg} / \mathrm{m}^{3}$, because of the small expected velocities.

To determine the boundary conditions, necessary to simulate the treated problem, the physiology of 
the heart has to be known. As it is known, during the healthy closed valve the blood flows from aorta to left and right coronary artery and also to smaller arteries to the whole body, but this is not the case of this study. On the other hand, when regurgitation occurs, the blood is also flowing from aorta to the LV, because of the valve leak. Therefore the inlet boundary condition (BC) has been placed on the aortic plane and the outlet $\mathrm{BC}$ on the ventricle and both coronary planes. Except for the healthy aortic valve simulation, where the exclusion of the outlet on the ventricle plane has been done, because there is no blood flowing into the LV. Position of the planes is shown on Fig. 3. To solve the presented governing equations for this problem Eqs. (1) to (3) the value of the $\mathrm{BC}$ are required, which have been determent on the base of average physiological values for human at rest [14] to [16] and are presented in Table 2.

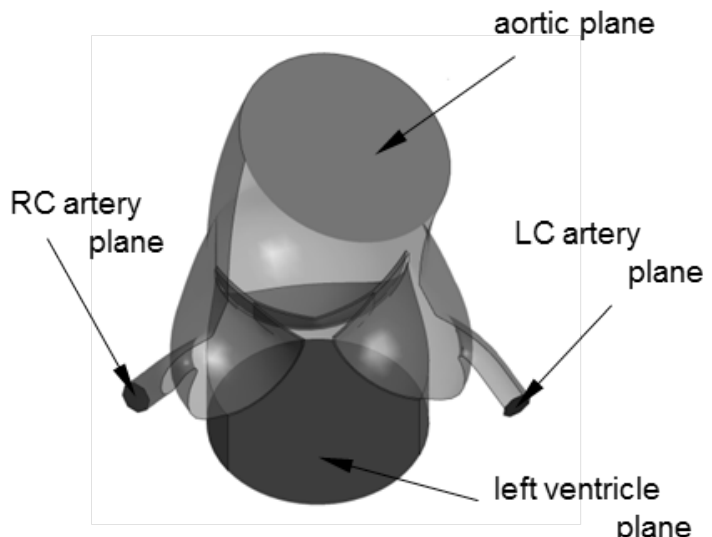

Fig. 3. Boundary condition planes position

Table 2. Parameters for definition of boundary conditions: $n_{S}$ the heart rate, $p_{A S}$ the maximal aortic pressure at systole, $p_{A D}$ the minimal aortic pressure at diastole, $Q_{L C}$ the blood flow through $L C$ artery, $Q_{R C}$ the blood flow through $R C$ artery and $t_{D}$ the diastole time

\begin{tabular}{|c|c|c|c|c|c|}
\hline \multicolumn{6}{|c|}{ Boundary condition parameters } \\
\hline $\begin{array}{c}n_{S} \\
{[1 / \mathrm{min}]}\end{array}$ & $\begin{array}{c}p_{A S} \\
{[\mathrm{kPa}]}\end{array}$ & $\begin{array}{c}p_{A D} \\
{[\mathrm{kPa}]}\end{array}$ & $\begin{array}{c}Q_{L C} \\
{[\mathrm{ml} / \mathrm{min}]}\end{array}$ & $\begin{array}{c}Q_{R C} \\
{[\mathrm{ml} / \mathrm{min}]}\end{array}$ & $\begin{array}{c}t_{D} \\
{[\mathrm{~s}]}\end{array}$ \\
\hline 75.0 & 15.93 & 10.62 & 190.0 & 40.0 & 0.5 \\
\hline
\end{tabular}

For the healthy aortic valve simulation the inlet $\mathrm{BC}$ with time-dependent relative aortic pressure has been prescribed to the aortic plane and its distribution is shown on Fig. 4. On the other hand the outlet BC placed on the $\mathrm{RC}$ and LC artery plane has been treated with prescribed mass flow rate. Coronary blood flow, which flows through coronary circulation (CC) is very complex, dependent on the pressure difference between RA and aorta, myocardium contraction, etc. Therefore the coronary mass flow rate has been described with linear equation:

$$
\dot{m}_{i}=\rho \frac{\Delta p_{i}}{R_{i}},
$$

where $\Delta p_{i}$ is a perfusion pressure and the resistance of $\mathrm{CC}$. The mass flow rate has been evaluated at the end of each time step for the next time step iteration. It is well known that the blood flow rate through coronary arteries is maximal during the diastole, because of the minimal $\mathrm{CC}$ resistance. The reason is because myocardium relaxes and the vessels dilatate, especially in endocardium. Therefore the CC resistance is time-dependent and it is shown on Fig. 5. Its distribution has been chosen, based on the normal coronary blood flow at rest, which is known. Some assumptions have been made here in setting the timedependent distribution of resistance, like linear change during the myocardium relaxation and contraction as well as constant value during ventricular filling. To go step further the $\mathrm{CC}$ resistance is also controlled with myocardium metabolism, which is higher in LV and consequently causes a higher blood flow rate in LC than RC artery. For this reason the resistance of right $\mathrm{CC}$ has been chosen to be smaller than resistance of right CC. Other mechanisms for resistance regulation are myogenic and neural, which has not been implemented in this study, because it exceeds the scope of this work. The perfusion pressure that occurs in Eq. (5) has been defined with

$$
\Delta p_{i}=p_{i c}-p_{R A},
$$

where $p_{i c}$ represents a pressure in the coronary plane and equal to the pressure in the ascending aorta and $p_{R A}$ represents the pressure in right atrium (RA). The reason is because the blood flows from aorta, through coronary arteries and $\mathrm{CC}$ to RA. The prescribed timedependent RA pressure in Eq. (6) is also shown on Fig. 4.

The $\mathrm{BC}$ for the regurgitated aortic valve simulation has been changed. The outlet $\mathrm{BC}$ at the $\mathrm{LC}$ and $\mathrm{RC}$ artery plane has been treated in the same manner as before with Eqs. (5) and (6). However because the mass flow rate is dependent from an aortic pressure, the mass flow drop has been expected owing to the decreasing pressure. Aortic pressure decreases because of the leaking blood back to the LV. This way the time-dependent relative pressure at the aortic plane has been defined and been calculated at each time step with equation: 


$$
p_{A}(t+\Delta t)=p_{A}(t)-\Delta p_{P}-\Delta p_{V}
$$

where $p_{A}(t+\Delta t)$ is the aortic pressure in the next time step, $p_{A}(t)$ is the pressure in the present time, $\Delta p_{P}$ is pressure drop because of the blood flow from aorta through systemic circulation (whole body) and $\Delta p_{V}$ isthe pressure drop because of the back flowing blood, that is from aorta to LV. Pressure drop $\Delta p_{P}$ has been defined from the aortic pressure distribution at healthy aortic valve (Fig. 4). On the other hand the pressure drop $\Delta p_{V}$ is not known in advance; therefore it has been described with equation:

$$
\Delta p_{V}=\frac{\dot{m} \Delta t}{\rho C},
$$

where $\dot{m}$ represent a mass flow into the LV (regurgitated blood flow), $\Delta t$ is a time step and $C$ aortic stiffness. The mass flow has been evaluated from the results at the end of each time interval. Therefore the pressure drop $\Delta p_{V}$ has been calculated by Eq. (8) at the end of each time step to update the relative aortic pressure in the next time step defined by Eq. (7). The value for aortic stiffness has taken to be $8.023 \mathrm{~m}^{3} / \mathrm{Pa}$ and can vary greatly, depends on the person age. To close the system of equations, also the outflow BC on the LV plane has to be defined. For this the time-dependent $\mathrm{LV}$ relative pressure has been prescribed and is also shown on Fig. 4. Because the chronic regurgitation of aortic valve has been taken into account, the increase in LV end diastolic pressure was neglected. Finally also the non-slip boundary condition has been prescribed for all the walls.

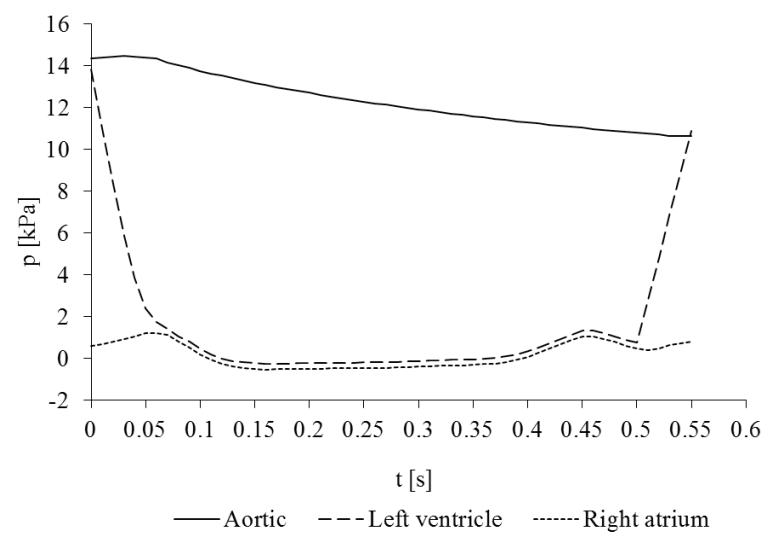

Fig. 4. Boundary conditions: aortic, LV and RA pressure

The problem has been treated as time-depended; therefore the initial value for the flow field needs to be defined. Therefore the initial values were defined with steady state simulation for initial boundary conditions.
For consistence of several figures and results the beginning of valve closure was set to $0 \mathrm{~s}$.

This way the $\mathrm{BC}$ at regurgitated aortic valve depends on the problem itself as well as the results of the simulation. In this manner it is possible to obtain the effect of regurgitation on the cardiac cycle as well as on the heart, like aortic pressure drop, increased volume of LV, the change of coronary blood flow, etc.

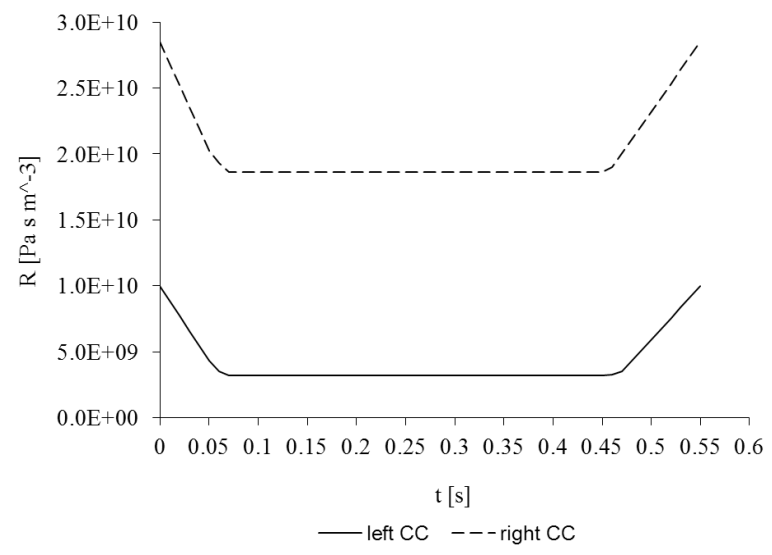

Fig. 5. Boundary conditions: resistance of left and right CC

\subsection{Properties}

Geometry of computational domain has been discretizated with tetrahedral and prism elements to create computational mesh. At the wall the inflation layer has been used for better description of velocity gradients. For regurgitated aortic valve simulation, the computational mesh has also been refined in the region of the central hole also to better describe the high velocity gradients. Representative mesh for healthy and regurgitated valve is shown on Fig. 6. The computational mesh has also been analysed to estimate the numerical error and to prevent inaccurate numerical results. The final mesh consists of 80,376 nodes and 275,046 elements for healthy aortic valve and of 83,180 nodes and 301,820 elements for regurgitation.

Numerical model also needs be time discretizated, because of the time-dependent problem. The proper time step has been set by comparing the results of two different time steps of the test case. Therefor the time step at healthy aortic valve simulation has been set to $0.025 \mathrm{~s}$ and for regurgitation to $0.001 \mathrm{~s}$.

The blood velocity during the diastole is assumed to be low enough to have laminar flow field and therefore the turbulence models have not been included in numerical models. The convergence criteria for solving the problem have been set to $10^{-4}$. 

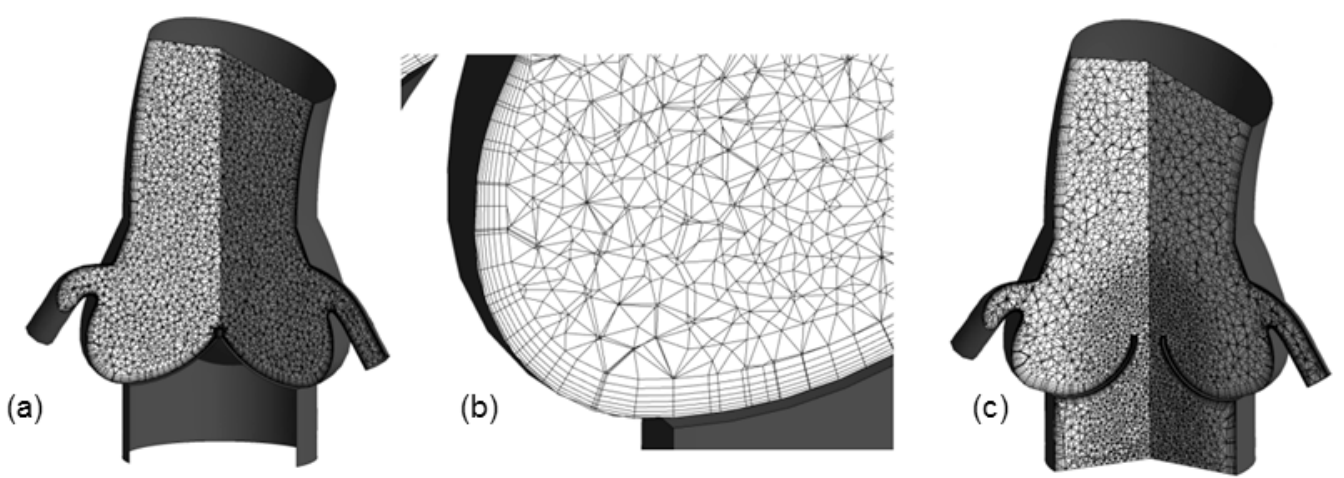

Fig. 6. Mesh for computational domain: a) healthy valve, b) detailed view of inflated layer and c) aortic valve regurgitation

\section{RESULTS AND DISCUSSION}

Transient simulation of blood flow through coronary arteries and around aortic valve, during the closed valve, for healthy and diseased (regurgitated) aortic valve, has been done. The duration of closed valve for healthy aortic valve has been set to $0.55 \mathrm{~s}$ as it can be seen on Fig. 4. Fig. 7 shows the blood velocity field around the healthy aortic valve for the time $0.15 \mathrm{~s}$, when the coronary blood flow is the highest. The velocity vectors have been normalized to clearly show the direction of the flow. As discussed earlier the blood flow rate through LC artery is substantially higher than through the RC artery, what can also be seen from Fig. 7. The average flow rate through the $\mathrm{RC}$ artery during the closed valve has been $36.48 \mathrm{ml} /$ min and through the LC artery $187.6 \mathrm{ml} / \mathrm{min}$. Even the maximal flow rate through $\mathrm{LC}$ artery is pointing to a low Reynolds number defined by:

$$
\operatorname{Re}=\frac{\bar{v} d \rho}{\bar{\eta}},
$$

where $\bar{v}$ represents average velocity, $\bar{\eta}$ average dynamic viscosity and the characteristic length which is in the diameter of the artery in this case. Maximal value of Reynolds number for LC artery has been 426.7 and for RC artery 42.3 , which indicates the laminar flow regime in the arteries and also in the region of aortic valve and support the assumption of excluding the turbulence models in the numerical models. As can be seen from Fig. 7, the blood movement around valve, this is cusps and lunulas, is also very small. First reason for that can be in low coronary blood flow and the other in high dynamic blood viscosity due to the non-Newtonian behaviour. Higher dynamic viscosity increases the resistance of moving and therefore small velocity field. Fig. 8 shows the dynamic viscosity of the blood through left and right cusp, which supports the above statement. As it can be seen the dynamic viscosity around aortic valve is higher, especially around right cusp, than from the origin of the coronary arteries.

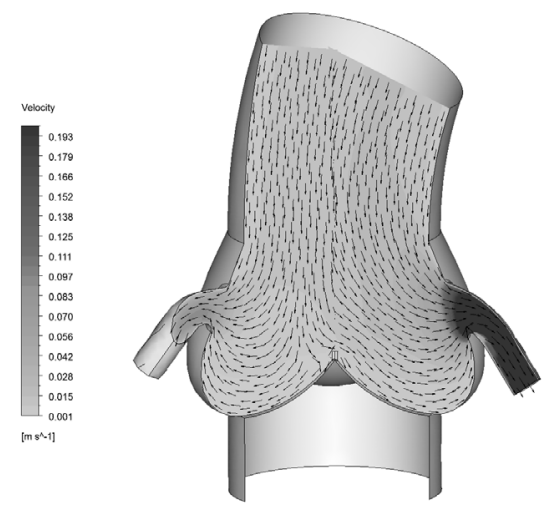

Fig. 7. Blood velocity field for healthy aortic valve $(0.15 \mathrm{~s})$

At aortic valve regurgitation simulation, the blood not only that flows from aorta to the left and right coronary artery but also from aorta through aortic valve hole to LV (regurgitated blood flow). The blood flow field can be seen on Fig. 9, which shows the flow filed at two different time 0.15 and $0.35 \mathrm{~s}$. As it is known this regurgitated blood flow decreases the aortic pressure by Eq. (7), which also gave the pressure drop as a result of simulation. This pressure drop in aorta is shown on Fig. 10, together with normal pressure drop at healthy aortic valve. If a comparison of the aortic pressure for regurgitated and healthy valve simulation is made, it can be seen that the drop is quite large. It can be also noticed that at regurgitation the valve stayed closed for only 0.52 $\mathrm{s}$, which is less than at the healthy valve. The reason lies in the difference between aortic and LV pressure, which is zero or even negative and therefore an opening of the aortic valve, begins. 


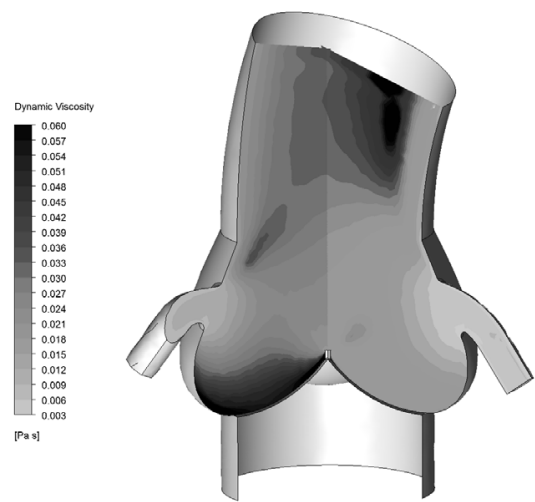

Fig. 8. Dynamic viscosity field for healthy aortic valve (0.15 s)

High aortic pressure drop is also causing the drop in the coronary blood flow. Fig. 11 shows the calculated time-dependent coronary blood flow for healthy and regurgitated aortic valve. For regurgitation, the average blood flow rate through $\mathrm{RC}$ artery has been $24.47 \mathrm{ml} / \mathrm{min}$, meanwhile through LC artery $132.55 \mathrm{ml} / \mathrm{min}$, which means a $31 \%$ drop.

With presented numerical models, the volume of regurgitated blood flow can also be calculated. For the threated regurgitation the volume of blood was $56.1 \mathrm{ml}$. That means that the LV is highly loaded with blood and that the regurgitation in those conditions can be severe. As it can be seen from the Fig. 9 also the strong jet occurs at the central hole. The maximum average velocity of the blood jet has been $3.7 \mathrm{~m} / \mathrm{s}$, which gave the Reynolds number of 6084 . Reynolds number has been evaluated by Eq. (9), where the hydraulic diameter of $3.8 \mathrm{~mm}$ has been taken as a characteristic length. The hydraulic diameter has been defined by equation:

$$
d=\frac{4 A}{u},
$$

where $A$ represent a cross sectional area and $u$ the wetted perimeter. High value of Reynolds number indicates the tendency of turbulent flow, but because of the small time of this phenomena and short distances, the question if the flow is really turbulent, needs some further research. However on the other hand at sever valve regurgitation the regurgitated blood flow is supposed to be turbulent; because it creates a murmur. The strong blood jet also produces the slow recirculation in LV as can be seen on Fig. 9. This recirculation is driven by the velocity of the jet and not by the continuum equation, as it would be if the computational domain would include the whole LV geometry. Therefore this recirculation, because of small computational domain and prescribed boundary conditions, is not physically correct. The strong central jet is also stressing the cusps around the central hole, because of the low pressure field under the cusps. This can also be shown with the shear stress on the surfaces, like it is shown on Fig. 12. Great shear stress on the tissue can cause the lesion or improper function of the tissue. As seen on the Fig. 12, the high shear stress in the region of the central hole tends to move the cusps together to close the central hole. Therefore, some error has been made using non-elastic geometry. For the same reasons as before, also in the regurgitated valve simulation it can be noticed, that the blood movement is slow around the valve cusps. Fig. 13 is showing the dynamic viscosity of the blood, which can be associated with the region of slowly moving blood. Also because the back flow occurs at the beginning of the valve closure,
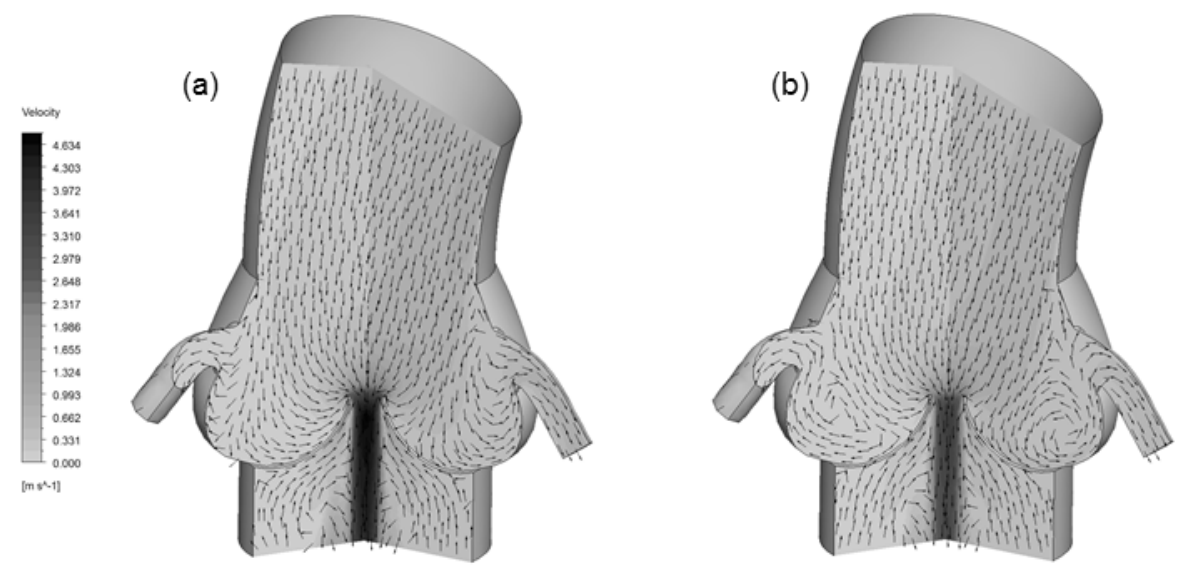

Fig. 9. Blood velocity field for aortic valve regurgitation; a) $0.15 \mathrm{~s}$, b) $0.35 \mathrm{~s}$ 
the error of using poor initials values for the flow field is assumed to be minimal.

The question arises, if the results from presented numerical models are precise enough, because some assumptions have been made. Like nonelastic geometry, this may be the most important one. To answer that question further investigation or improvement of numerical model should be done. The improvement to use elastic geometry will lead to the whole cardiac cyclic simulation and the error of using poor initial values for flow filed would be excluded. One of the promising methods to incorporate fluidstructure interaction and elastic geometry with high distortion could be meshless particle method described by Petkovšek et al. [17]. Second most important improvement would be to investigate the behaviour and the impact of different non-Newtonian blood models. Also more detailed investigation of important factors that affects the coronary blood flow should be done. This would lead to the improved mathematical model Eq. (5), because the current model has a disadvantage in the $\mathrm{CC}$ resistance. This has to be evaluated in advanced, based on the known coronary blood flow. Physically more accurate model would be, if the resistance would be a function of the metabolic, myogenic and neural regulation. As well the model for evaluation of aortic pressure drop because of the regurgitated blood flow Eq. (8) could be improved, to compensate the LV parameters like, LV volume and its pressure rising. These few improvements are stated here just to remind the reader what assumptions have been made and how the evaluation of the results in the further research should be done.

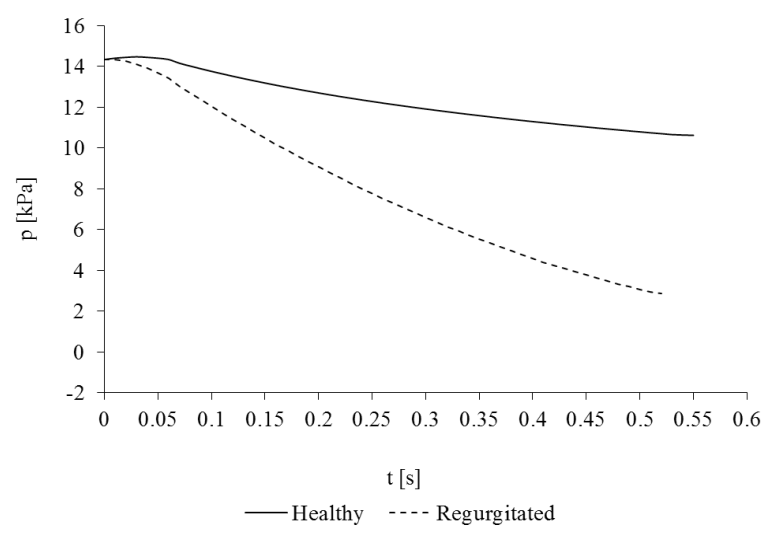

Fig. 10. Aortic pressure for healthy and regurgitated aortic valve

Simulation of blood flow around healthy as well as regurgitated aortic valve shows that the blood is moving real slowly around the leaflet and sinuses. This slow motion can cause a deposition of calcium or calcification and with that correlated aortic valve stenosis. The other factor that helps calcium deposition is the deposition time or time the valve being closed, which is longer for resting position than for exercise. Reason for calcification that is stated here is just an assumption, which should need some further investigation.

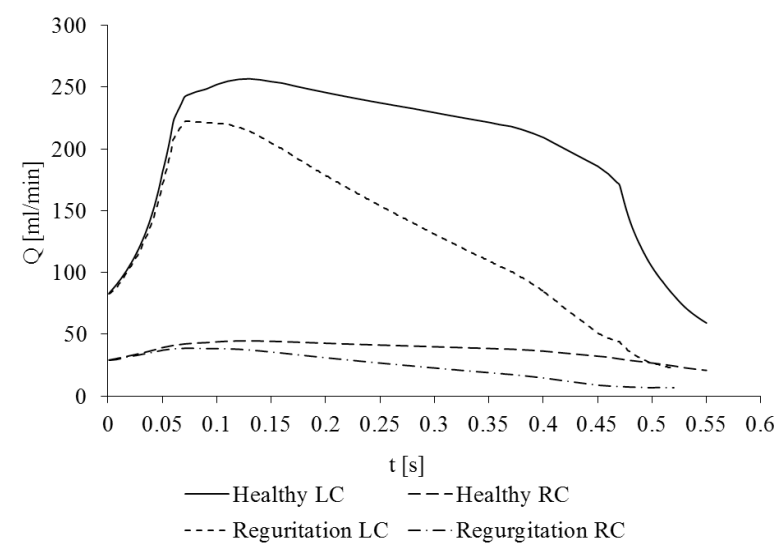

Fig. 11. Coronary blood flow during closed valve for healthy and regurgitated aortic valve simulation

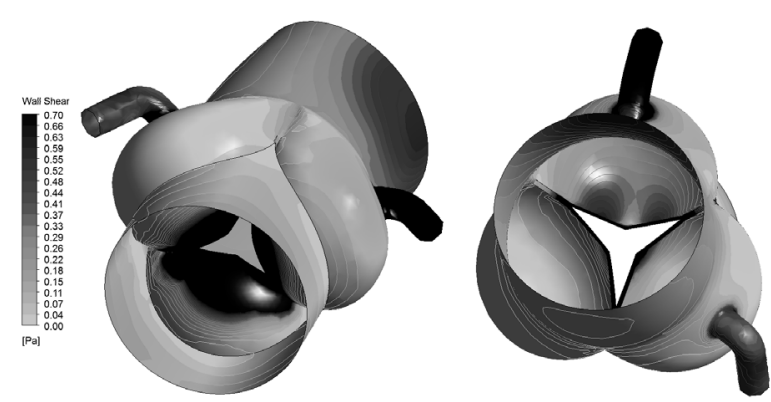

Fig. 12. Wall shear stress in aortic valve regurgitation

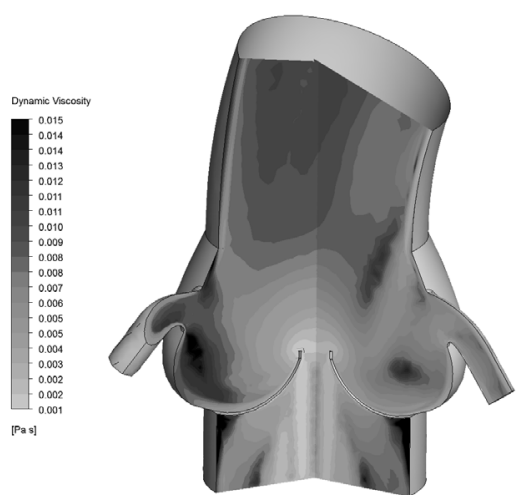

Fig. 13. Dynamic viscosity field for regurgitated aortic valve (0.35s)

De Heart et al. [8] have also done the simulation of the blood wash-out, which indicates that the blood 
particles around the leaflets does not stays in that position longer than two cardiac cycles. The most important assumption in De Heart simulation has been the use of blood as Newtonian fluid, which may lead to the mislead results. As well the coronary blood flow has been neglected. As it can been seen in this study the effect of coronary blood flow on the blood around aortic valve and sinuses was minimal, so the assumption of excluding the coronary arteries may be reasonable. However the effect of coronary blood flow can be substantial during the exercise, where the coronary blood flow can be up to four times larger.

Simulation of chronically regurgitated aortic valve has shown that the LV is highly loaded, because of the large regurgitated blood volume. Therefore the effective volume of blood, which had to be pumped from LV each cardiac cycle, has increase severely. For this reason the work done by myocardium has also been increased as well as the demand of the oxygen. However on the other hand the supply of the oxygen has been reduced as shown on Fig. 11, which is because of the pressure drop in ascending aorta. Of course this is not happening in a real life, because other mechanisms, like vasodilatation, etc. came in place to reduce the resistance of $\mathrm{CC}$ and to help maintain the demand and supply in balance. But in long term, these mechanisms cannot cope with oxygen demand and myocardial ischemia occurs. In this study only the drop of the coronary blood flow rate at the same $\mathrm{CC}$ resistance has been investigated. Nevertheless for these conditions the coronary blood flow drop at $7.7 \%$ regurgitation is quite severe. As stated before, for more precise calculation of coronary blood flow drop, the new mathematical model would be needed.

\section{CONCLUSION}

This study shows the difference between the healthy and regurgitated biological aortic valve, based on presented numerical model. It covers how the regurgitation influences the coronary blood flow, aortic pressure drop, volume load of the LV, cardiac cycle, etc.

The computational domain has been narrowed to the aortic valve region with origin of the coronary arteries and its geometry has been covered in detail. However some geometric assumptions have been made, the created geometry still represents normal biological valve and its region. The reason lies in fact that the geometry of the aortic valve distinguishes from person to person.
The physical problem has been treated as timedependent and solved only for the period of closed valve. The reason for that lies in the nature of regurgitation and coronary blood flow. Therefore the assumption of using non-elastic geometry has been made. In addition the blood was treated as an incompressible, pseudo plastic non-Newtonian fluid with the use of Power Law model. To solve the physical problem or to close the system of governing equations, the boundary conditions have been applied to the boundary of computational domain, like aortic pressure, LV pressure and mass flow through LC and $\mathrm{RC}$ artery. The domain has also been geometric and time discretizated and to evaluate the accuracy of discretization the nodalization analysis have been done. Mass flow through coronary arteries has been described with mathematical model, Eq. (5) as well as aortic pressure drop at regurgitation, Eq. (7). This way the coronary blood flow and also aortic pressure drop have not been prescribed in advance, but are the results of numerical solution. Therefore the comparison between healthy and regurgitated aortic valve can be done.

This study shows that regurgitation can highly load the LV; also the oxygen delivery can be greatly decreased, which can lead to myocardium ischemia. Therefore the aortic valve dysfunction, like regurgitation, can have a serious impact on heart function as well as on the human health. Furthermore the coronary blood flow shows little or no affect on the blood movement around the valve and in the ascending aorta. However the question arise what happens during the exercise, when the coronary blood flow increases. At that point the possible reason for calcification has also been stated, but needs some further investigation. The main reason for the slow blood movement around the valve cusps and correlated calcification is in the nature of the blood itself or its dynamic viscosity, which indicates that using the blood as a Newtonian fluid will lead to misleading results.

Discussion also covers the further improvements of numerical model, like using elastic geometry and evaluation of the results using different nonNewtonian blood models.

With the help of diseased aortic valve simulation, the disease impact on the cardiac cycle as well on the heart function can be evaluated. As well as the evaluation, when the disease progresses too much, that the heart cannot cope with it anymore and urgent surgery is needed. However for this situation, the numerical model would have to be improved, what represents the future work on this study. 


\section{REFERENCES}

[1] Lai, Y.G., Chandran, K.B., Lemmon, J. (2002). A numerical simulation of mechanical heart valve closure fluid dynamics. Journal of Biomechanics, vol. 35, no. 7, p. 881-892, DOI:10.1016/S0021-9290(02)00056-8.

[2] Krafczyk, M., Cerrolaza, M., Schulz, M., Rank, E. (1998). Analysis of 3D transient blood flow passing through an artificial aortic valve by Lattice-Boltzmann methods. Journal of Biomechanics, vol. 31, no. 5, p. 453-462, DOI:10.1016/S0021-9290(98)00036-0.

[3] De Hart, J., Peters, G.W.M., Schreurs, P.J.G., Baaijens, F.P.T. (2000). A two-dimensional fluidstructure interaction model of the aortic valve. Journal of Biomechanics, vol. 33, no. 2, p. 1079-1088, DOI:10.1016/S0021-9290(00)00068-3.

[4] De Hart, J., Peters, G.W.M., Schreurs, P.J.G., Baaijens, F.P.T. (2003). A three-dimensional computational analysis of fluid-structure interaction in the aortic valve. Journal of Biomechanics, vol. 33, no. 1, p. 103112, DOI:10.1016/S0021-9290(02)00244-0.

[5] De Hart, J., Baaijens, F.P.T., Peters, G.W.M., Schreurs, P.J.G. (2003). A computational fluid-structure interaction analysis of a fiber-reinforced stentless aortic valve. Journal of Biomechanics, vol. 36, no. 5, p. 699712, DOI:10.1016/S0021-9290(02)00448-7.

[6] De Hart, J., Peters, G.W.M., Schreurs, P.J.G., Baaijens, F.P.T. (2004). Collagen fibre reduce stresses and stabilize motion of aortic valve leaflets during systole. Journal of Biomechanics, vol. 37, no. 3, p. 303-311, DOI:10.1016/S0021-9290(03)00293-8.

[7] Carmody, C.J., Burriesci, G., Howard, I.C., Patterson, E.A. (2006). An approach to the simulation of fluidstructure interaction in the aortic valve. Journal of Biomechanics, vol. 39, no. 1, p. 158-169, DOI:10.1016/j.jbiomech.2004.10.038.

[8] Johnston, B.M., Johnston, P.R., Corney, S., Kilpatrick, D. (2004). Non-Newtonian blood flow in human right coronary arteries: steady state simulations. Journal of Biomechanics, vol. 37, no. 5, p. 709-720, DOI:10.1016/j.jbiomech.2003.09.016.

[9] Johnston, B.M., Johnston, P.R., Corney, S., Kilpatrick, D. (2006). Non-Newtonian blood flow in human right coronary arteries: Transient simulations. Journal of Biomechanics, vol. 39, no. 6, p. 1116-1128, DOI:10.1016/j.jbiomech.2005.01.034.

[10] Boutsianis, E., Dave, H., Frauenfelder, T., Poulikakos, D., Wildermuth, S., Turina, M., Ventikos, Y., Zund, G. (2004). Computational simulation of intracoronary flow based on real coronary geometry. European journal of Cardio-thoracic Surgery, vol. 26, p. 248256, DOI:10.1016/j.ejcts.2004.02.041.

[11] Labrosse, M.R., Carsten, J.B., Robicsek, F., Thubrikar, M.J. (2006). Geometric modeling of functional trileaflet aortic valves: Development and clinical applications. Journal of Biomechanics, vol. 39, no. 14, p. 2665-2672, DOI:10.1016/j.jbiomech.2005.08.012.

[12] Fuster, V., Aleksander, R.W., O'Rourke, R. (eds.). (2001). Hurst's The Heart, tenth edition. McGraw-Hill, New York.

[13] Ternik, P., Žunič, Z., Marn, J. (2007). Blood hemodynamics in carotid bifurcation: influence of rheological models. IWNMNNF: Book of abstracts, Rhodes.

[14] Berne, R.M., Levy, M.N. (2000). Principles of physiology, third edition. Mosby, London.

[15] Bronzino, J.D. (ed.). (2006). Biomedical engineering fundamentals, third edition. Taylor \& Francis Group, New York.

[16] Skalak, R., Chien, S. (eds.). (1987). Handbook of bioengineering. McGraw-Hill, New York.

[17] Petkovšek, G., Džebo, E., Četina, M., Žagar, D. (2010). Application of Non-Discrete Boundaries with Friction to Smoothed Partile Hydrodynamics. Strojniški vestnik - Journal of Mechanical Engineering, vol. 56, no. 5, p. 307-315. 